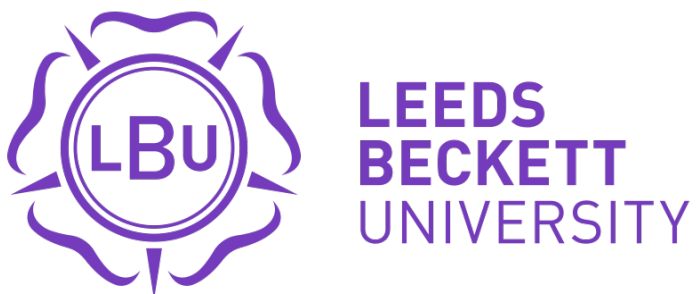

\section{Citation:}

Chang, V and Ramachandran, M (2017) Financial Modeling and Prediction as a Service. JOURNAL OF GRID COMPUTING, 15 (2). pp. 177-195. ISSN 1570-7873 DOI: https://doi.org/10.1007/s10723017-9393-3

Link to Leeds Beckett Repository record:

https://eprints.leedsbeckett.ac.uk/id/eprint/3667/

Document Version:

Article (Accepted Version)

The aim of the Leeds Beckett Repository is to provide open access to our research, as required by funder policies and permitted by publishers and copyright law.

The Leeds Beckett repository holds a wide range of publications, each of which has been checked for copyright and the relevant embargo period has been applied by the Research Services team.

We operate on a standard take-down policy. If you are the author or publisher of an output and you would like it removed from the repository, please contact us and we will investigate on a case-by-case basis.

Each thesis in the repository has been cleared where necessary by the author for third party copyright. If you would like a thesis to be removed from the repository or believe there is an issue with copyright, please contact us on openaccess@leedsbeckett.ac.uk and we will investigate on a case-by-case basis. 


\title{
Quality of Service for Financial Modeling and Prediction as a Service
}

\author{
Victor Chang and Muthu Ramachandran \\ School of Computing, Creative Technologies and Engineering, Leeds Beckett University, Leeds, UK. \\ \{V.I.Chang; M.Ramachandran\}@leedsbeckett.ac.uk
}

Keywords: Quality of Service (QoS) for SaaS; Financial Modeling and Prediction as a Service (FMPaaS) QoS; performance and accuracy test for FMPaaS QoS.

\begin{abstract}
This paper describes our proposal for Quality of Service (QoS) for Financial Modeling and Prediction as a Service (FMPaaS), since a majority of papers does not focus on SaaS level. We focus on two factors for delivering successful QoS, which are performance and accuracy for FMPaaS. The design process, theories and models behind the FMPaaS service have been explained. To support our FMPaaS service, two APIs have been developed to improve on performance and accuracy. Two major experiments have been illustrated and results show that each API processing can be completed in 2.12 seconds and 100,000 simulations can be completed in an acceptable period of time. Accuracy tests have been performed while using Facebook as an example. Three points of comparisons between actual and predicted prices have been undertaken. Results support accuracy since results are between $93.72 \%$ and $99.63 \%$.
\end{abstract}

\section{INTRODUCTION}

The complexity of large scale financial cloud computing services that require high speed and high precision systems grows exponentially. Services of large scale financial cloud computing and grids are enormous in recent years. Some of them are used for weather forecasting, simulation of aircraft and military services, atmospheric and planet study, remote sensing, large scale data analysis, aerospace research, large scale computational fluid dynamic services, aeronautics and automobile industries, and financial simulations. More recently, predication models used by these applications have become increasingly important (Cantor and Royce, 2014). As a result, understanding the behavioral aspects of such systems is important for the design in the quality of service. Some characteristics of large scale financial cloud computing services include:

- High speed and highly parallel

- Real-time

- Virtually connected nodes of systems
- Grid is an infrastructure for large scale financial cloud computing and other resources

- High precision and accuracy

To manage largely-scale software in the cloud, software components and also known as service components are used. The aim is to provide a selfcontained entity that can be adapted to the required environment quickly and easily. To elaborate this further, software components design for large scale financial cloud computing and grids have become major issues in recent years and in years to come (Silvestri et al., 2006; Albodour et al., 2012). They have all claimed the importance of software components which will dominate large scale financial cloud computing and grid services. Albodour et al. (2012) propose a model, Business Grid Quality of Service (BEQoS), to measure key metrics and provide added value for commercial and business Grid applications. They use the GridSim software to demonstrate their proof-of-concepts with supporting results to show that reliability and affordability can be achieved. Silvestri et al (2006) assert that the future large scale financial cloud computing and grid services can be completely built 
in a bottom-up fashion using software components deployed on various locations and interconnected to form a workflow graph and to re-configure themselves as and when needed during run-time to self manage those services that may in need.

In this paper, we propose a QoS requirements engineering model to assert certain subsets of activities that must be identified and assessed for a large scale financial cloud computing and grid services where the main emphasis has been given to non-functional requirements that match onto the characteristics of such Services. In all the applications and Software as a Service (SaaS), financial applications require on-demand services that are offered by cloud computing with costbenefits. Hence, financial domain has begun to reap this benefit with emerging financial SaaS such as FinancialForce developed jointly by SalesForce, NetSuite, Intacct, and Oracle's financial SaaS. According to NetSuite (2014), FinancialForce helped companies increase their revenues by $95 \%$. Accenture (2011) reports on financial technology trends and high performance computing prediction in the following category:

- Leveraging technology to address new \& change in regulations

- Reliable and globally harmonized financial systems

- Add value through strategic applications

- Harvest benefits from technology

According to Accenture (2011), SaaS should be simple, efficient, engaging, accessible, clearly structured, intuitive, and supportive. While keeping this set of requirements as design criteria, a SaaS component model and a service architecture should be designed to support flexibility, scalability, and adaptability. This paper has proposed an integrated service-oriented architecture and SaaS component model for financial domains which provides required scalability, flexibility and customization that are at the heart of a financial SaaS.

There are a number of QoS factors that affect quality of a cloud service. We have proposed a set of QoS attributes that are keys to success of cloud services, in particular, Financial Modeling and Prediction as a Service (FMPaaS) where accuracy and performance are the key benefits of such services which has been achieved. To demonstrate accuracy, two types of the accuracy test were given. The first type was focused on the overall accuracy and the second type was focused on three point selection. One example will be illustrated to support accuracy for our FMPaaS.

\subsection{QoS for Financial Modeling and Prediction as a Service (FMPaaS)}

Cloud is committed to providing everything as a service and QoS can provide multiple parameters that are required by financial cloud computing services. There are a number of QoS metrics to be considered for FMPaaS. In our previous work (Chang, 2014), we demonstrated the use of FMPaaS in business intelligence applications and identified six important factors. The importance of each factor can be measured in the scale between 1 and 10. A complete set of QoS factors that affects FMPaaS are identified in Figure 1 and some which have been validated in our earlier project on FMPaaS (Chang, 2014) and are summarized as follows:

- Usability: Most of QoS APIs are easy to use except one API requires further training. The overall score is 8 because at least $80 \%$ of the tools are easy to use and their manuals are selfexplanatory. The other $20 \%$ of the functionalities require specialized knowledge about financial modeling to compute complex models.

- Performance: Performance on QoS is good. Computation takes a short time to get results. The score is 8 .

- Security: QoS needs third party software and is not a model with a high level of security. Basic authentication and authorization can still be achieved. As a result, the score is 4.

- Computational accuracy: Computational QoS results are accurate. Some banks have used QoS to calculate pricing and risks, and are close to the actual values. But QoS requires have accurate input values before getting the final results. This level of dependency is a limitation to prevent it to score 10 . The overall score is 8 .

- Portability: QoS is highly portable in most of the systems. All operating systems and computational devices can run QoS applications. The overall score is 9 .

- Scalability: QoS tools are highly scalable. It can run on a single processor desktop, or clusters of high-end servers. Input variables can be highly adaptable to a wide range of values.

These scores for QoS are based on the results of expert reviews of eleven experts. Follow-up improvements are required to support the QoS model. In addition to these well know parameters to measure QoS, we have also defined a clear model and equation to measure QoS in terms of satisfaction of services on the fly. We highlight important factors essential for QoS success, with more emphasis paid on performance and accuracy. Referring to Figure 1, a list of QoS parameters are used in our work to 
evaluate service quality. We highlight important factors essential for QoS success, with more emphasis paid on performance and accuracy.

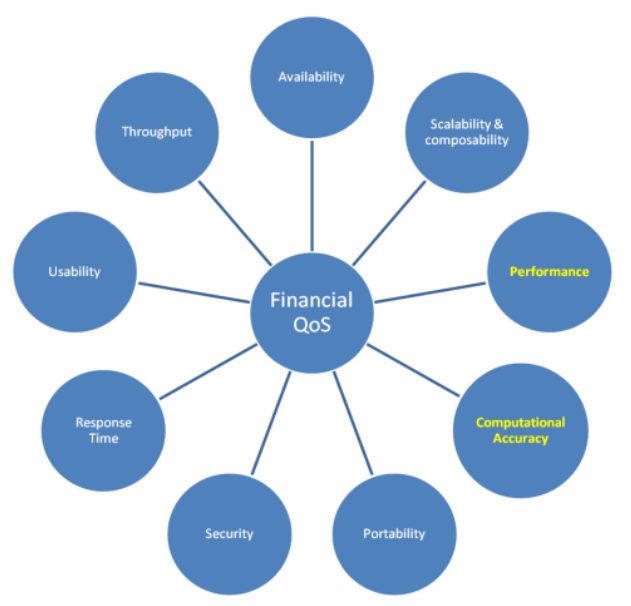

Figure 1: QoS Metrics to Measure

\subsection{Our approach in QoS for Financial Modeling and Prediction as a Service (FMPaaS)}

In review of all the six factors influencing QoS, we have already demonstrated the importance of security in our papers (Ramachandran and Chang, 2014). In this paper, we will elaborate on these factors, in particular performance and accuracy. The reasons are as follows. First, literature presented in Section 1.1 does not provide details in accuracy. While SaaS is essential to sectors such as finance and medicine which require an extremely high level of accuracy, any errors or glitch may cause damaging impacts. If FMPaaS calculates incorrect results such as advising investors to buy a particular stock with millions of pounds, or a reliable stock at a particular instance with millions of pounds, they can bear the consequence. This means that the emphasis in QoS accuracy is essential for Cloud Computing.

Second, there is an increased demand to offer accurate predictive services, since the inaccurate results may cause financial loss, loss of company reputation, loss of consumer confidence. This is a type of QoS that have not been presented in the research computing community. For example, if they lose out million of pounds due to the misleading predictive results from similar FMPaaS services, it may result in bankruptcy (Lehman Brothers), loss of reputation (UBS) and loss of investors apart from the direct loss of money. Similarly, simulations related to human bodies such as brain, heart and vital organs are important to determine the most likely scenarios for patients receiving treatments for several years.

With regard to FMPaaS, one of our contributions to $\mathrm{QoS}$ is the notion of service satisfaction index which can be in-built as part of a service specification. FMPaaS index allows users evaluate services based on their merits in real scenarios and also supports service reusability, a key benefit of service computing. In reviewing all factors contributing to QoS success, we focus more on accuracy and performance to ensure that our FMPaaS can provide as correct and swift as possible for investors. We emphasize on the software design approach for FMPaaS QoS and use one example to illustrate our proof-of-concepts.

\section{INANCIAL MODELING AND PREDICTION AS A SERVICE QOS}

This section describes the system design for Financial Modeling and Prediction as a Service (FMPaaS) QoS, which is essential in a few disciplines. For example, e-government applications require open, flexible, interoperable, collaborative and integrated architecture to provide services. These services can be made available as stand alone, integrated, componentized, web based service component, composite service (a set of interconnected services), virtualized services (cloud based), and dynamically re-configurable services. This vision is similar to the Open Group's (2009) Service Integration Maturity Model (OSIMM), which provides:

- A process roadmap for attaining key practices with metrics

- Seven levels of maturity to improve

- A quantitative model for assessing current practices and to improve with recommended practices

As mentioned earlier section, service components are useful to manage system complexity and reuse of services during autonomous service composition. The key challenge is to design a service component that supports service characteristics discussed earlier. A service component can be defined as a self autonomous service which provides two sets of services: provider business services and required business services. The provider business service (often shown with a lollypop notation and the naming convention starts with I) is a set of services offered to other services to compose where as the required business services 
(often shown as a semi-arc notation) are a set of services that are required by this service in order to compose successfully. In this work, we have proposed a component model for FMPaaS applications as shown in Figure 2, which the required services include Income statement, ICashFlow statement, Ie-taxation, IFSA regulations. IFSA provides interface service integration for Financial Authority regulations. Ay investment service providers can integrate their work to this FMPaaS service component model, which is adaptable to regular updates in regulations. By doing so, FMPaas can provide scalability and flexibility for financial analysts. These services can be made available as stand alone, integrated, componentized, web based service component, composite service (a set of interconnected services), virtualized services (cloud based), and dynamically re-configurable services.

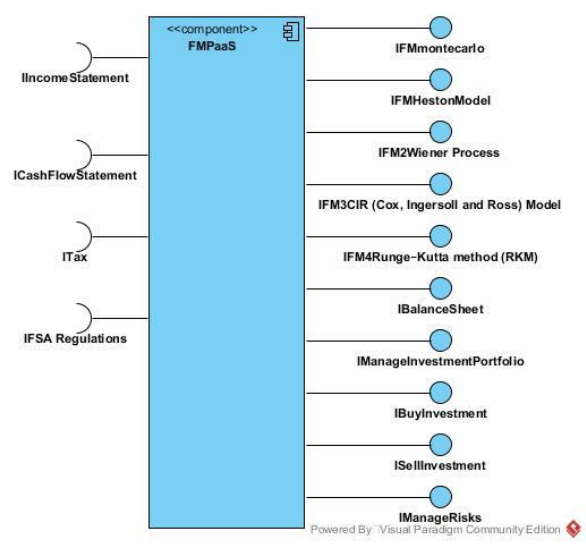

Figure 2: FMPaaS Service Component Model

The next step in the design process is to design service-oriented cloud architecture for FMPaaS where all aspects of the corporate financial service are integrated and composed based a set of SLA and governance. The architecture presented in this paper is based on a critical review and analysis of a number of existing architectures for FMPaaS applications. Further to this, the SOA based architecture consists of four distinct levels of abstraction layers which are connected and communicated by messages through a core communication channel known as a service bus or a central bus. These layers are: 1) a business layer with a dedicated set of services; 2) an orchestration layer with a set of services where new services can be composed; 3) an FMPaaS layer that supports integration of services, government departments and local governments, and 4) an e-business layer that supports new businesses and integration of data. The SOA based architecture for FMPaaS services, then ensures that it achieves the expected service-oriented design factors such as customization, costeffectiveness, availability, etc. The service-oriented FMPaaS architecture is shown in Figure 3.

Referring to Figure 3, at the business and orchestration layers provide high level service composition based on new business perspective and policies (both political and economical factors). Mostly, the customization and the new business needs arise from these two key variables. The subsystems such as registration control, security control, integrated services for FMPaaS applications control, and communications channels help to achieve customization at a higher level of abstraction without affecting underlying business logic services. These are communicated and connected to layers below using a concept of service bus known as FMPaaS secured service bus. The layer below the business layer provides services for various FMPaaS departments, and external suppliers (E-Business layer). Software components for large scale financial cloud computing services require a detailed analysis of the domain and its boundary in order to define a collection of components for large scale financial cloud computing services that are highly reusable and scalable. A good SaaS design should introduce a domain analysis process which allows us to define a set of common definitions, domain classification, domain boundaries, domain models, design artifacts, and design guidelines that are based on those domain criteria.

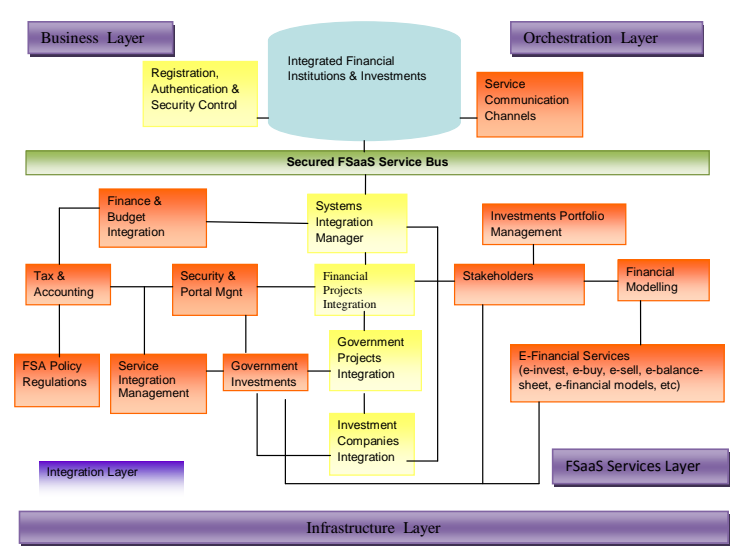

Figure 3: Service-Oriented Architecture for FMPaaS 


\section{MODELS AND THEORIES BEHIND FMPAAS}

The current work on QoS (Lee et al, 2009; Mukhopadhyay, 2012; Shehu et al., 2014) have proposed a number of frameworks and are useful in its own merits. However, they only have an emphasis on other non-functional attributes and then claim non-functional attributes as QoS parameters. Similar to Albodour et al. (2012), our proposed model is to provide commercial uses for research institutes, financials services and general public who are involved or interested in stock market analysis. The main difference between our work and Albodour et al. (2012) is that we use our own development of work. We have developed a comprehensive approach based on the development of FMPaaS extended from our current work, which aims to distinguish QoS attributes clearly; helps to identify them from requirements to model financial cloud and then validate services against those attributes. These include the followings:

1. Based on the reputable models - the chosen model is the Heston Model (which includes the Wiener process and the Stochastic Volatility) and the Visualization APIs to compute the best pricing and risks for different scenarios.

2. Accuracy to compute and track volatility FMPaaS can track the movement of volatility and help investors make a better judgment for investment when prices are high and volatility is low. Our FMPaaS can compute pricing and risk values to several decimal places and also calculate its mean, lower and upper range to get our results as accurate as possible.

3. Performance - all calculations should be completed within seconds to ensure all services can be delivered in an acceptable time frame.

\subsection{Models used for FMPaaS}

Models behind FMPaaS are essential for the calculation, processing and presentation of financial computation in the Cloud. Our previous work explains all the associated models, including the choice of the models, their associated formulas, how they can be used in the development of FMPaaS. In summary, models include (Chang, 2014):

1. Heston Model

2. Wiener Process

3. CIR (Cox, Ingersoll and Ross) Model

4. Runge-Kutta method (RKM)

The use of all the models for FMPaaS can match accuracy and optimize the performance. The summary of their descriptions is as follows.

\subsubsection{The Heston Model}

The Heston Model has a close relationship with Black-Scholes model, since it relaxes the constant volatility assumption in the classical Black-Scholes model by incorporating an instantaneous short term variance process (Albrecher et al., 2006). In other words, the Heston Model can be used in a more flexible way and is not as theoretical-oriented as the classical Black-Scholes model does. In addition, there are both the Wiener process and the CIR process related to the Heston Model. Heston Model has been explained in our previous work and it can still be very useful for undertaking business intelligence services and prediction of financial modeling (Chang, 2014).

\subsubsection{The Heston Model}

The Wiener process is a stochastic process with independent and stationary increments, which means the motion of a point whose consecutive displacements are independent and random with each other. The Wiener process has Lévy characterization has continuous martingale with W0 $=0$ and quadratic variation $[\mathrm{Wt}, \mathrm{Wt}]=\mathrm{t}$. This implies that Wt2-t is a martingale (Cox et al., 1985; Kloeden and Platen, 1999). The basic Heston model assumes that $S_{t}$, the price of the asset, is determined by a stochastic process (Cox et al., 1985; Kloeden and Platen, 1999). The Heston Model has a CIR process involved, which is a Markov process with continuous paths defined by the following stochastic differential equation (SDE). The variable include Wiener process (i.e., random walks) with correlation $\rho \mathrm{dt}$. The parameters in the Heston model for providing input in the computation in Section 4 represent the following:

- $\mu$ is the rate of return of the asset.

- $\theta$ is the long variance, or long run average price variance; as $t$ tends to infinity, the expected value of $v_{t}$ tends to $\theta$.

- $\kappa$ is the rate at which $v_{t}$ reverts to $\theta$.

- $\xi$ is the volatility of the volatility; as the name suggests, this determines the variance of $v_{t}$.

\subsubsection{The CIR Model}

The CIR process is used to model stochastic volatility in the Heston model, which aims to resolve a shortcoming of the Black-Scholes model which corresponds to the fact that the implied volatility does tend to vary with respect to strike price and expiry. By assuming that the volatility of the underlying price is a stochastic process rather than a constant, stochastic volatility can make it possible to 
model derivatives more accurately (Cox et al., 1985; Wilmott and Wilmott, 2006).

\subsubsection{The Runge-Kutta method}

The Runge-Kutta method (RKM) is a technique for the approximate numerical solution of a stochastic differential equation (SDE) (Hull and White, 1987; Wilmott, 2006). RKM can be used to generalize the ordinary differential equation to SDE. To elaborate further, the Ito diffusion $X$ satisfying the following Ito stochastic differential equation (Hull and White, 1987; Wilmott and Wilmott, 2006). Details of formulations can be referred to Chang (2014).

\subsection{Methods for FMPaaS calibration}

This section describes methods for FMPaaS calibration, which is used in a way that a known observation of the dependent variables is used to predict a corresponding explanatory variable. The root-mean square error (RMSE) and Moving Window (MW) are identified as the methods to perform FMPaaS calibration.

\subsubsection{The root-mean square error}

The root-mean square error (RMSE) is used to measure of the differences between values predicted by a model or an estimator and the values actually observed. RMSE also determines the goodness of fit of the Heston Model presented by Cox et al. (1985) and Hull and White (1987).

$$
R M S E=\sqrt{\frac{\sum_{i=1}^{n}\left(X_{o b s, i}-X_{\text {model }, i}\right)^{2}}{n}}
$$

where $\mathrm{n}$ is the number of quoted options, $X_{o b s}$ is observed values and $X_{\text {model }}$ is modelled values at time/place $i$. The parameters required for RMSE include $\left(v_{0}, \kappa, \theta, \xi, \rho\right)$ used for calibration and $v_{0}$ is the instantaneous variance at the starting point. Referring to formula (2), the rate of return of the asset can be calculated by multiplying $\kappa$ and difference between $\theta$ and $v_{0}$.

\subsubsection{The Moving Window}

The Moving Window (MW) estimate is a suitable model in the use of VIX options, which are provided daily to track market values of volatility. MV can be computed as the mean of variance of the stock price process over the time series window that moves forward in time. MW is used to compute the forecasted movement in the Heston Model.

\subsubsection{Average absolute percentage error (APE) and aggregated relative percentage error (ARPE)}

The average absolute percentage error (APE) of the mean price and aggregated relative percentage error (ARPE) are additional formulas for calibration to construct the best fit in financial computation, and thus improves the accuracy and performance of the calculations (Wilmott, 2006; Kloeden, and Platen, 2012; Guillaume and Schoutens, 2012). A limitation with APE is that it may cause a problem. A few of the series with a very high APE might distort a comparison between the average APE of time series fitted with one method compared to the average APE when using another method. To overcome this limitation, another model, aggregated relative percentage error (ARPE) is used.

\subsection{Services on offer}

This section explains two types of services on offer for FMPaaS QoS. The architecture adopts the private cloud at the University of London Computing Centre (ULCC) data center and Southampton clusters, where the processing took place in ULCC. Two types of services are as follows.

- Heston Volatility and Pricing as a Service (HVPaaS): The request started and completed at Southampton clusters, including the processing of the HVPaaS. The objective is to track volatility and pricing simultaneously since both can change significantly during the volatile period. The metrics are provided by the respective inputs of Heston model except volatility, which is provided by VIX.

- Business Analytics as a Service (BAaaS): After analyzing the numerical computation of volatility and pricing, the next step is to compute them as a Business Analytic. This makes the analysis much easier and the stakeholders can understand. After the processing of HVPaaS completed in Southampton, results are sent to ULCC in London, where both sites can process BAaaS. This service is regarded as the case of a complete FMPaaS QoS. 
Application Programming Interfaces (APIs) are used to illustrate how to use these two services. In BAaaS, it has two APIs as follows.

1. FinancialData API - this allows the BIaaS Cloud to obtain financial data from Google Finance and have all the major stock market data, particularly the US and UK stock exchange data.

2. TradingChart API - this allows the financial data to be presented in the trading chart format similar to the visualization services offered by London Stock Exchange and Thomson Reuters. Additional functions can allow analysts to use the MW model to compute forecasted movement. "TradingChart" is the API to demonstrate both models (Heston and Financial data) can work together to deliver an integrated service. Results of the experiments will be presented in Section 4.

\subsection{Measurement of FMPaaS QoS}

This section describes the measurement of FMPaaS QoS, which aims to demonstrate the significance of performance and accuracy. In terms of performance, the execution time for all APIs should be recorded to check their completion time is within seconds. Experiments involved with multicore and multi-node processing are included to illustrate the performance issue. To demonstrate accuracy, an approach is to compare the predicted result from the FMPaaS QoS with the actual results generated by the market such as the New York Stock Exchange or London Stock Exchange. The end results of these APIs, particularly the TradingChart API (the last one of all FMPaaS services), can correspond to the predicted results of the FMPaaS analysis. The actual results can be imported directly from Google Finance. The difference between the actual and predicted results can correspond to the percentage of accuracy. The objective is to maintain all differences within 5\% difference to ensure a high quality of accuracy to be achieved.

\section{ACCURACY TESTS AND RESULTS OF PERFORMING FMPAAS QOS SIMULATIONS}

This section describes the accuracy tests of the selected stocks listed on the New York Stock Exchange. Some of these selected stocks are the continuation of our previous study which analyzed stocks between mid-May 2012 and early July 2013. Hence, we will analyze the stocks between early July 2013 and mid-May 2014. Additionally, some of the new selected stocks such as Citi and GE are used to analyze the accuracy of FMPaaS results. Our previous work has shown the stocks of Facebook, Apple, IBM and Microsoft between mid-May 2012 and end of June 2013 and these four stocks are used again for FMPaaS analysis (Chang, 2014).

\subsection{The Overview of the FMPaaS}

This section presents the overview of the FMPaaS, including the end results of the analysis shown in Figure 4. The first section of Figure 4 is the main area of FMPaaS QoS, where the y-axis shows the price and the $\mathrm{x}$-axis shows the time scale. There are upper and lower lines, which are predicted indexes based on the stock values every ten minutes ago. As explained in innovative business intelligence (Chang, 2014), both upper and lower limits offer $95 \%$ of confidence interval (CI) for the predictive modeling. The line in the middle is the predicted value line based on the values given 10 minutes ago and without using the $95 \% \mathrm{CI}$ approach. The second section represents the trading volume, the quantity for buys and sales. The third section represents the relative strength index, which means how active the stock movement is compared to 50 as the baseline. In this case, we are only focused on the first section, the accuracy and performance of the actual and predicted index movements since the correctness of prediction can affect the quality of sales and buys.

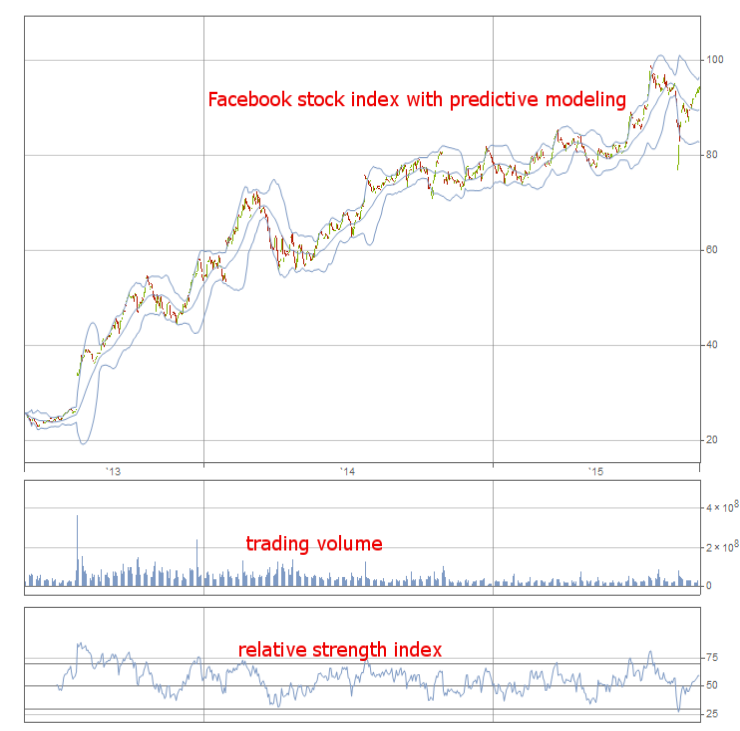


Figure 4: The full FMPaaS result showing Facebook stock prices, volume and relative strength between 18 May, 2013 and 26 September 2015

\subsection{Performance test: The experiments with APIs}

As explained in Section 3.3, development of APIs is essential for FMPaaS to measure the effectiveness of QoS. Our previous work also demonstrates the use of two APIs, "FinancialData" and "TradingChart", which display the outputs of FMPaaS based on the calculation and computation of formulas presented in Section 3. The outputs measure the following two items:

- The status of the return, which are the prices of the assets at the times that sales are intended;

- Volatility, which represent the variable market risk associated with the sale or buy activities.

Experiments with these two APIs are important since they will determine the performance of generating results and accuracy of the results received. To present the results of experiments, the hardware specifications are described in Section 4.2.1. Steps and processes involved with two experiments are then presented in Section 4.2.2 and 4.2 .3 respectively.

\subsubsection{Infrastructure used for experiments}

University of London Computer Center (ULCC) was used for the experiments. ULCC has advanced Cloud and parallel computing infrastructure and network attached storage (NAS) service. It has 10 quadcore CPUs, each with $3.0 \mathrm{GHz}, 60 \mathrm{~GB}$ of RAM and $12 \mathrm{~TB}$ of disk space for experiments. Fiber optic network offering the $10 \mathrm{~Gb}$ network speed was used for experiments. The network was connected to the first private clouds based at Greenwich, which has a total of 3 quadcore of CPUs with $3.0 \mathrm{GHz}$ each and 20 GB RAM. The infrastructure at ULCC is also connected to the second private cloud based at the University of Southampton, which have 3 quadcore of $3.0 \mathrm{GHz}$ each and $16 \mathrm{~GB}$ RAM in place. There is the third private cloud based at the author's venue at Southampton, which has the capability is 8 quadcore CPUs with $3.0 \mathrm{GHz}$ each and $32 \mathrm{~GB}$ RAM. All the three private clouds located in Greenwich and two places at Southampton have been connected to ULCC through the fast fiber optic networking and the VMWare infrastructure. Before experiments took place, preliminary work had been tested and all the outputs could be successfully computed. The distance between different private clouds did not make a difference in the execution time during the preliminary phase of the experiments.

\subsubsection{Execution time for a single API processing}

This section presents results of processing each API in two settings. The first experiment was undertaken between the two private clouds at Southampton. The second experiment was undertaken while utilizing both the Southampton and ULCC clouds. In other words, results should be sent to ULCC for processing and returned back to Southampton. The execution time is the total time of processing mathematical modeling on the APIs on the server and response time to the client. The first experiment was expected to take less time due to the shorter distance. All experiments were conducted five times with the mean values taken as the execution time and the standard deviation was the difference between the highest and lowest values. The results of API experiments were presented in Table 1.

Table 1: The execution time for each API or process in the local environment $(\mathrm{p}<0.005)$

\begin{tabular}{|c|c|c|}
\hline API or process & $\begin{array}{c}\text { Southampton } \\
\text { execution time } \\
(\mathrm{sec}) \text { and } \\
\text { standard } \\
\text { deviations }\end{array}$ & $\begin{array}{c}\text { ULCC: execution } \\
\text { time (sec) and } \\
\text { standard } \\
\text { deviations }\end{array}$ \\
\hline FinancialData & $2.04(0.10)$ & $2.12(0.12)$ \\
\hline TradingChart & $1.11(0.03)$ & $1.19(0.06)$ \\
\hline
\end{tabular}

\subsubsection{Execution time for 100,000 simulations of API processing}

Results in Section 4.2.2 show the average execution time of one simulation per API processing. To test the performance, the large-scale simulations are required (Guillaume and Schoutens, 2012). Our FMPaaS can offer up to 100,000 simulations per service to test the scenarios that if there are 100,000 service requests happen every second, whether our FMPaaS can still provide services smoothly without degrading the service. The aim of this experiment is to demonstrate that our FMPaaS can support 100,000 service requests and achieve a good execution time. Availability was $100 \%$ at the time that those experiments were taken, with the network and VMs working in excellent conditions. All the experiments were taken five times with the mean values taken as the execution time and the standard deviation was the difference between the highest and lowest values. Results are presented in Table 2 . 100,000 simulations on the API could be completed 
in 200,645 seconds, or 55 hours, 44 minutes and 5 seconds.

Table 1: The execution time for 100,000 simulations of API processing in the ULCC $(\mathrm{p}<0.005)$

\begin{tabular}{|c|c|c|}
\hline API or process & $\begin{array}{l}\text { Southampton } \\
\text { execution time } \\
(\text { sec }) \\
\text { and standard } \\
\text { deviations }\end{array}$ & $\begin{array}{c}\text { ULCC: execution } \\
\text { time (sec) and } \\
\text { standard } \\
\text { deviations }\end{array}$ \\
\hline FinancialData & $200432(488)$ & 200645 (499) \\
\hline TradingChart & $110135(417)$ & $110348(429)$ \\
\hline
\end{tabular}

All the standard deviations are below $0.5 \%$ of the average execution time for all six APIs. The aim for this experiment is to demonstrate that in the event of having 100,000 requests from users in realtime, how the FMPaaS can respond to all the processing. Results also show that FMPaaS can cope with 100,000 requests.

\subsection{Accuracy test}

This section describes the accuracy test by using Facebook as an example to illustrate. The focus is to demonstrate accuracy and performance of using FMPaaS analysis. The execution time of performing this FMPaaS test is 3.15 seconds, which correspond to the sum of processing "FinancialData" and "Tradingchart" APIs. We identify three major points where the predicted asset prices would be compared directly with the actual prices. The reason was that since price values could change all the times, identifying the points for comparison was useful. Additionally, this can ensure prediction to be more focused on the end of the trading activities since they could receive more investors' attention.

Two types of accuracy tests are presented. The first test is focused on the overall level of accuracy, whether all the actual values fall into the upper and lower predicted values within the range of $95 \%$ confidence interval (CI). The second test is based on three selection points where the trading activities are at the end of the quarterly business review, or at three obvious points in the FMPaaS result. In Figure 5 , points 1,2 and 3 are chosen due to the location of these points to be checked and noticed easily.

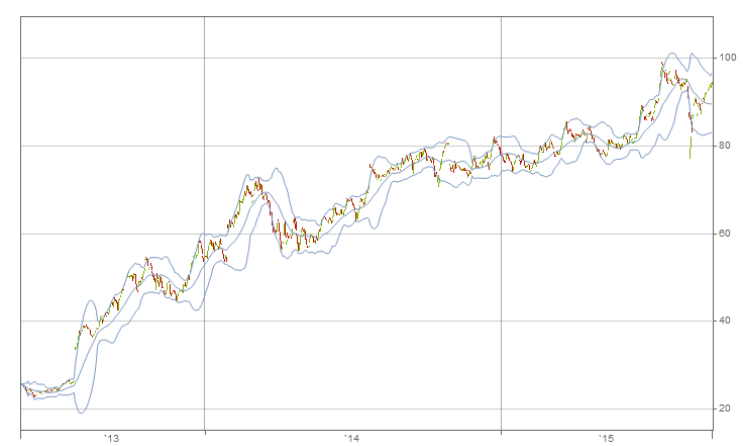

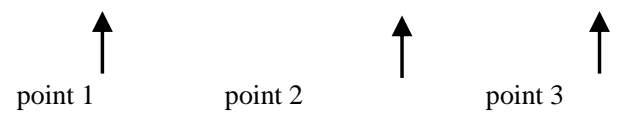

Figure 5: The FMPaaS result showing Facebook stock prices between 2 July, 2013 and 16 May 2014

Table 3 shows the results of the overall accuracy test. We count the number of datapoints falling outside the $95 \%$ CI divided by the total number of datapoints. The results show that about $97 \%$ of the actual datapoints, or actual values of Facebook index movements, fall within the $95 \%$ CI predictive range. Among those $3 \%$ falling outside the predicted range, there is one spot with a red arrow. This happened because Facebook was reported to have more profits than their analysts' forecasted results. However, the market had the mixed reactions in the first few days, which resulted in numerous selling and buying activities. Those who bought thought that Facebook would have a better value at some point. Those who sold thought that it was a time to get their investment back. This explains why our forecasted values slightly deviate from the actual values. Additional calibration can be used to compute the forecast price values and volatility for the three points, where the results can then be used to compare with the actual values for the accuracy.

Table 3: The test of the overall accuracy for Facebook

\begin{tabular}{|c|c|c|c|}
\hline Items & $\begin{array}{c}\text { Falling } \\
\text { within } \\
95 \% \text { CI } \\
\text { lines }\end{array}$ & $\begin{array}{c}\text { Percentag } \\
\text { e falling } \\
\text { outside } \\
95 \% \text { CI } \\
\text { lines }\end{array}$ & $\begin{array}{c}\text { Significant spots } \\
\text { falling outside } \\
95 \% \text { CI lines }\end{array}$ \\
\hline Actual \\
values & $\begin{array}{c}\text { Yes. 97\% } \\
\text { of actual } \\
\text { values are } \\
\text { within the } \\
\text { range. }\end{array}$ & About 3\% & $\begin{array}{c}\text { Profits were } \\
\text { more than their } \\
\text { predicted results } \\
\text { between } \\
2013 / 2014 \\
\text { forecast. }\end{array}$ \\
\hline
\end{tabular}

To determine the accuracy test, asset prices of the predicted values (input values by Heston model and VIX and computed by models in Section 3) are directly compared with the actual values. See Table 4 for results. Asset prices computed by the predicted value are close to their respective actual values in points 1, 2 and 3, ranging between $93.72 \%$ and $99.63 \%$ accuracy. Points 2 and 3 have extremely high accuracy and point 1 has an acceptable level of accuracy. The likely reason is that the asset price prior reaching point 1 was on the way up to one and 
a half months and it was less predictable to forecast the asset price values on the way up in point 1 .

Table 4: The test of the three selection point accuracy for Facebook

\begin{tabular}{|c|c|c|}
\hline Items & Actual value & Predicted value \\
\hline $\begin{array}{l}\text { Point } \\
1\end{array}$ & $\begin{array}{l}\text { Asset price = } \\
\text { 50.15; volatility = } \\
1.20 ; \quad \text { implied } \\
\text { volatility = } 0.45 ; \\
\text { time }=0.3\end{array}$ & $\begin{array}{l}\text { Asset price }=\mathbf{4 7 . 0 0} \text {; } \\
\text { volatility }=1.20 ; \\
\text { implied volatility }= \\
0.45 ; \text { time }=0.3 \text {. } \\
\mathbf{9 3 . 7 2 \%} \text { same as the } \\
\text { actual value }\end{array}$ \\
\hline $\begin{array}{l}\text { Point } \\
2\end{array}$ & $\begin{array}{l}\text { Asset price = } \\
\text { 53.30; volatility }= \\
0.5 ; \quad \text { implied } \\
\text { volatility = } 0.45 ; \\
\text { time }=0.6\end{array}$ & $\begin{array}{l}\text { Asset price = 53.70; } \\
\text { volatility }=0.5 \text {; implied } \\
\text { volatility }=0.45 ; \text { time }= \\
0.6 .99 .26 \% \text { close to } \\
\text { actual value }\end{array}$ \\
\hline $\begin{array}{l}\text { Point } \\
3\end{array}$ & $\begin{array}{l}\text { Asset price }= \\
\mathbf{5 9 . 0 1 ;} \text { volatility = } \\
0.5 ; \quad \text { implied } \\
\text { volatility = } 0.35 ; \\
\text { time }=1.15\end{array}$ & $\begin{array}{l}\text { Asset price = 59.23; } \\
\text { volatility }=0.5 ; \text { implied } \\
\text { volatility }=0.35 ; \text { time }= \\
1.15 .99 .63 \% \text { same as } \\
\text { the actual value }\end{array}$ \\
\hline
\end{tabular}

\subsection{Discussion}

The benefits of adopting FMPaaS are as follows. First, FMPaas have focused on improving the accuracy for the financial modeling and prediction as demonstrated in the test results. This can also provide new and alternative services for forecasting and investment analysis. Second, FMPaaS can provide positive impact to the stakeholders and potential investors to understand the market performance, volatility, trading volume and likely predicted movements of their chosen stocks. These two aspects of contributions will help the stakeholders, potential investors and research community to understand the market much better. The benefits offered by FMPaaS are relevant to the themes of Emerging Software as a Service and Analytics to allow the community to know an improved and better Cloud SaaS services being validated and illustrated with reported contributions. The next phase of challenges is to improve the overall level of accuracy from $95 \%$ to $98 \%$ and above; improve the point accuracy as close as to $99.99 \%$ and raise three points of evaluation and testing to six points to ensure there is a greater coverage of accuracy tests.

\section{CONCLUSION AND FUTURE WORK}

A large number of QoS papers focus on the hardware infrastructure and Service Level Agreement with the lack of explanation and further development for SaaS. We explain the motivation and significance of QoS for FMPaaS, which is our main service for finance and business intelligence. Six factors for delivering FMPaaS QoS have been illustrated, where the emphasis for this paper is on performance and accuracy. We first start with the design process and methodology for FMPaaS, and then explain the theories behind FMPaaS. APIs are provided in the FMPaaS, where "FinancialData" and "TradingChart" are the two APIs that have been developed and then used in the experiments for performance tests. Two types of experiments were conducted. First, each API was tested five times top get the mean execution time to generate outputs. All execution time was completed within 2.12 seconds. Second, large scale of 100,000 simulations was performed to test whether APIs can provide realtime services. Results show that 100,000 simulations on the API could be completed in 200,645 seconds, or 55 hours, 44 minutes and 5 seconds with a low percentage of standard deviations. Accuracy had been conducted to test the differences between the predicted and actual values. Three points of comparisons for Facebook stock were used for accuracy test since they represented the end of all transaction activities. Results show that accuracy tests had between $93.72 \%$ and $99.72 \%$ of accuracy while comparing the actual and predicted values of the asset prices of Facebook stock. Our future work will include the improvement of our performance and accuracy tests. We will also use more companies to illustrate that our FMPaaS can provide better services and accuracy while comparing the actual and predicted values of asset prices.

\section{REFERENCES}

Accenture, 2011, Accenture Financial Trends slides, http://www.slideshare.net/fullscreen/ramblingman/acc enture-financial-saa-s-external-presentation-final/3, accessed on April 2014

Albodour, R., James, A., N. Yaacob, 2012, High level QoS-driven model for grid applications in a simulated environment. Future Generation Computer Systems, 28(7), 1133-1144.

Albrecher, H., Mayer, P., Schoutens, W., and Tistaert, J., 2006, The Little Heston Trap, Technical paper, September. 
Cantor, M. and Royce, W., 2014, Economic Governance of Software Delivery, IEEE Software, 31(1).

Chang, V., 2014. The business intelligence as a service in the cloud. Future Generation Computer Systems, 37, 512-534.

Cox, J.C., Ingersoll J.E. \& Ross, S.A. 1985, A Theory of the Term Structure of Interest Rates, Econometrica 53: 385-408.

Durrett, R., 2000, Probability: theory and examples, 4th edition. Cambridge University Press, ISBN 0-52176539-0.

Guillaume F., and Schoutens, W., 2012, Calibration risk: Illustrating the impact of calibration risk under the Heston model, Review of Derivatives Research, 15:57-79.

Hull, J., and White, A., 1987, The Pricing of Options on Assets with Stochastic Volatilities, The Journal of Finance, 42(2).

Lee, J. Y., Lee, J. W., Cheun D. W. \& Kim S. D., 2009, QoS A Quality Model for Evaluating Software-as-aService in Cloud Computing, the Seventh ACIS International Conference on Software Engineering Research, Management and Applications.

Kloeden, P.E, Platen, E., 1999, Numerical Solution of Stochastic Differential Equations. Berlin: Springer. ISBN 3-540-54062-8.

Mukhopadhyay, D., Chathly, F. J., Jadhav, N. N., 2012, QoS Based Framework for Effective Web Services in Cloud Computing, Journal of Software Engineering and Applications, 5, 952-960.

NetSuite, 2014, white paper and software, product http://www.netsuite.co.uk/portal/uk/seo-landingpage/accounting $-2 /$ main.shtml?gclid=CLK9k $5 \mathrm{q}-$ 37sCFTHLtAodikoAzw, accessed on April.

Open Group, OSIMM, 2009, from https://www2.opengroup.org/ogsys/jsp/publications/P ublicationDetails.jsp?publicationid $=12450$, Retrieved Oct 2013.

Ramachandran, M., Chang, V., 2014 Cloud Security proposed and demonstrated by Cloud Computing Adoption Framework, the first international workshop on Emerging Software as a Service and Analytics, Barcelona, Spain, 03 - 05 April.

Schulze, B., Coulson, G., Nandkumar, R., Henderson, R., 2006, Special Issue: Middleware for Grid Computing: A 'possible future', Concurrency and computation: practice and experience, 10.1002/cpe.1132, Wiley.

Shehu, U., Epiphaniou, G., Safdar, G. A., 2014, A Survey of QoS-aware Web Service Composition Techniques, International Journal of Computer Applications (0975 -8887), 89(2), March.

Silvestri, F. et al., 2006, Toward a search architecture for software components, Journal of Concurrency and Computation: Practice and Experience: 18:13171331.

Wilmott, P., 2006, Paul Wilmott on quantitative finance, Wiley (2nd ed.), ISBN 0470018704. 